\title{
A comida de rua no Centro Histórico de Cartagena, Colômbia: consumidores, práticas e percepções
}

\author{
Street food in the Historic Center of Cartagena, Colombia: consumers, practices and perceptions \\ Comida callejera en el centro Histórico de Cartagena, Colombia: consumidores, practicas y
} percepciones

Claudia Patricia Alvarez Contreras

ORCID: https://orcid.org/0000-0002-1944-407X Universidade Federal da Bahia, Brasil E-mail: claupalcon@hotmail.com

Ryzia de Cassia Vieira Cardoso ORCID: https://orcid.org/0000-0001-5292-713X Universidade Federal da Bahia, Brasil E-mail: ryziac@gmail.com

Rafael Emilio González Cuello

ORCID: https://orcid.org/0000-0002-2674-2876 Universidade de Cartagena, Colômbia

E-mail: rgonzalezc1@unicartagena.edu.co

Clemente Granados Conde

ORCID: https://orcid.org/0000-0002-3201-4357

Universidade de Cartagena, Colômbia

E-mail: clementecondeg@gmail.com

Denise Nunes Viola

ORCID: https://orcid.org/0000-0001-8458-9489 Universidade Federal da Bahia, Brasil E-mail: deniseviola@gmail.com

Lis Nery Nunes da Silva

ORCID: https://orcid.org/0000-0003-0538-5926 Universidade Federal da Bahia, Brasil E-mail: lisnnsilva@gmail.com

Karina Castro Pomares

ORCID: https://orcid.org/0000-0001-7880-3899 Universidade de Cartagena, Colômbia E-mail: ingepaz2020@gmail.com

\begin{abstract}
Resumo
A comida de rua é um fenômeno mundial, notando-se insuficiência de estudos com consumidores. Assim, este estudo buscou caracterizar as práticas de consumo e a percepção de higiene e riscos de consumidores de comida de rua, no Centro Histórico de Cartagena, Colômbia. Realizou-se estudo transversal, com aplicação de questionário, junto a 90 consumidores. Na amostra, predominaram homens (70\%), com idade até 24 anos $(32,2 \%)$ e ensino superior completo (46,6\%). Para 70\%, o consumo da comida de rua ocorria desde a infância; $57,7 \%$ adquiriam-na, pelo menos, semanalmente, revelando o hábito. Os principais motivos para aquisição foram o fácil acesso $(41,1 \%)$ e o gosto/prazer $(38,3 \%)$, com maior probabilidade de consumo $(0,929)$ por mulheres de menor escolaridade. Frituras $(51,1 \%)$ e frutas $(26,6 \%)$ foram os alimentos mais consumidos - houve maior probabilidade do consumo de frituras por mulheres, menores de 24 anos, com baixa renda (0,8733), e do consumo de frutas por homens, maiores de 60 anos $(0,8502)$. Para 94,4\%, os alimentos poderiam oferecer riscos à saúde; $52 \%$ confiam "às vezes" na qualidade e higiene das frutas. Para $81 \%$, a venda de comida de rua gerava trabalho e renda. O estudo revela a importância alimentar e social do segmento, demandando atenção pública para seu melhor funcionamento.
\end{abstract}

Palavras-chave: Práticas alimentares; Setor informal; Segurança alimentar; Riscos sanitários.

\section{Abstract}

Street food is a worldwide phenomenon, with a lack of studies on consumers being noted. Thus, this study sought to characterize consumption practices and hygiene and risks perception among street food consumers in the Historic Center of Cartagena, Colombia. A cross-sectional study was carried out, with the administration of a questionnaire, along with 90 consumers. In the sample, there was a predominance of men (70\%), aged up to 24 years (32.2\%), with complete higher education $(46.6 \%)$. For $70 \%$, the consumption of street food occurred from childhood; $57.7 \%$ acquired it at least weekly, revealing the habit. The main reasons for purchase were easy access (41.1\%) and 
taste/pleasure $(38.3 \%)$, with higher probability of consumption $(0.929)$ by women with less education. Fried foods $(51.1 \%)$ and fruits $(26.6 \%)$ were the most consumed foods - there was a higher probability of fried foods by women, under 24 years old, with low income $(0.8733)$, and of fruit consumption by men, older than 60 years $(0.8502)$. For $94.4 \%$, street food could offer health risks; $52 \%$ trust "sometimes" in the quality and hygiene of the fruit. For $81 \%$, the sale of street food generated work and income. The study reveals the food and social importance of the sector, which demands public attention for its better functioning.

Keywords: Foodways; Informal sector; Food security; Health risks.

\section{Resumen}

La comida callejera es un fenómeno mundial, destacándose pocos estudios con consumidores. Así, este estudio buscó caracterizar las prácticas de consumo y la percepción de higiene y riesgos de los consumidores de alimentos en la vía pública en el Centro Histórico de Cartagena, Colombia. Se realizó un estudio transversal, con aplicación de cuestionario, con 90 consumidores. En la muestra, hubo predominio de hombres (70\%), de hasta 24 años $(32,2 \%)$ y con estudios en nivel superior completo (46,6\%). Para el 70\%, el consumo de comida callejera ocurrió desde la niñez; El 57,7\% lo adquirió al menos semanalmente, revelando el hábito. Los principales motivos de compra fueron el fácil acceso $(41,1 \%)$ y el gusto / placer $(38,3 \%)$, con mayor probabilidad de consumo $(0,929)$ por parte de mujeres con menor nivel educativo. Los alimentos fritos $(51,1 \%)$ y las frutas $(26,6 \%)$ fueron los alimentos más consumidos, existiendo una mayor probabilidad de consumo de frituras por parte de mujeres, menores de 24 años, con bajos ingresos (0,8733), y de consumo de frutas por hombres, mayores de 60 años (0,8502). Para el 94,4\%, los alimentos pueden presentar riesgos para la salud; El 52\% confía "a veces" en la calidad e higiene de las frutas. Para el $81 \%$, la venta de comida callejera generó trabajo e ingresos. El estudio revela la importancia alimentar y social del segmento, demandando atención pública para su mejor funcionamiento.

Palabras clave: Prácticas alimentarias; Sector informal; Seguridad alimentaria; Riesgos para la salud.

\section{Introdução}

A comida de rua representa parte importante da dieta de muitos habitantes nos países em desenvolvimento (FAO, 2011; Hiamey \& Hiamey, 2018). Estima-se que 2,5 bilhões de pessoas em todo o mundo consomem esse tipo de alimento todos os dias, representando até 30\% das compras domiciliares urbanas na América Latina (Mensah et al., 2013). Este é um segmento que tem mostrado crescimento contínuo ao longo dos anos, tornando-se essencial e popular para grande parte da população, principalmente nas áreas urbanas (Abrahale et al., 2019; Wiatrowski et al., 2021)

Devido à sua acessibilidade, baixo custo, variedades de produtos e proximidade do consumidor, a comida de rua passou a atender um público maior, reduzindo a demanda dos serviços de restaurantes, considerados principais fontes de alimentação fora de casa (Ayo et al., 2012). Além da conveniência e da disponibilidade, esses alimentos contribuem, significativamente, para a economia e para a preservação do patrimônio sociocultural, refletindo elementos da cultura alimentar local. A oferta de alimentos costuma variar ao longo do ano, de acordo com a safra, e depende do poder aquisitivo e das preferências culturais do público. Nesse sentido, significam aporte nutricional para os indivíduos que os consomem (Durán-Agüero et al., 2018; Hill et al., 2019)

Além da importância como acesso e consumo de alimentos, grande atenção neste setor se volta para a segurança e para o valor nutricional dos alimentos comercializados. Conforme pesquisas, há uma preocupação marcante quanto à segurança dos alimentos e as práticas de higiene nos vendedores. Todavia, esse é apenas um dos desafios, no que tange ao comércio e ao consumo dessas comidas, nos países em desenvolvimento (Alimi, 2016; Cortese et al., 2016; Diz et al., 2020).

Na Colômbia, país latino culturalmente marcado pela diversidade da comida de rua, essas características também se expressam no segmento. Além disso, muitas vezes, os vendedores de comida de rua operam sem regulamentação, devido à insuficiência de políticas públicas direcionadas ao setor (Contreras et al., 2020). Deste modo, à medida em que aumenta o número de pessoas que consomem esses alimentos, também aumenta a exposição potencial desses consumidores a riscos de origem alimentar (Adam et al., 2014; Hyun-Ah Kim; 2018).

De acordo com estudo global sobre Tendências de alimentação Fora de Casa, conduzido em 61 países - sete deles na América Latina, com levantamento online, a maioria dos entrevistados relatou comer fora do lar. Dentre esses, $15 \%$ dos 
Colombianos afirmaram consumir comida de rua (Nielsen, 2016).

Outrossim, independentemente da condição social, os colombianos comem na rua regularmente, atribuindo esse hábito ao tempo ou, simplesmente, ao capricho do que se apresenta diante dos olhos, motivos que os leva a ignorar os riscos sobre o modo de preparo da comida de rua. Apenas $28,5 \%$ dos colombianos afirmaram estar cientes dos riscos associados com a comida de rua, o que pode ser justificado, em parte, pela ocorrência de doenças decorrentes do consumo destes alimentos (El Espectador, 2012).

Na literatura, a preocupação dos consumidores com a inocuidade da comida de rua e os riscos associados não é uma questão bem definida, havendo estudos nos quais a confiança é baixa (Cardoso et al., 2017; Liu et al., 2014), enquanto em outros se observa bom nível de confiança (Asiegbu et al., 2015). Nesse contexto, é conhecido que a segurança desses alimentos pode ser afetada por vários fatores, como: a qualidade dos alimentos crus, as condições de preparo, de manuseio e de armazenamento, bem como a infraestrutura dos locais, que não atendem a todos os requisitos legais. Assim, de modo a proteger a saúde, há a necessidade de atenção e cuidado dos consumidores, na escolha dos alimentos e dos locais de aquisição da comida de rua (Alimi, 2016; Mwove et al., 2020).

Considerando a presença marcante da comida de rua na Colômbia e o papel essencial dos consumidores na prevenção de doenças transmitidas por alimentos e na redução de riscos, este estudo teve como objetivo conhecer as práticas de consumo e a percepção de higiene e riscos dos consumidores deste segmento, no Centro Histórico de Cartagena de Índias, Colômbia.

\section{Metodologia}

\subsection{Coleta de dados}

Foi realizado um estudo transversal, de abordagem quantitativa, junto a indivíduos que compravam alimentos prontos para o consumo, vendidos nas ruas do Centro Histórico de Cartagena de Índias, Colômbia. Trata-se de uma das principais cidades turísticas da Colômbia, que está localizada ao norte do departamento de Bolívar, na costa do Mar Caribe (10² 25' 30" N e $75^{\circ} 32^{\prime} 25^{\prime \prime} \mathrm{W}$ ). Com base em pesquisa realizada previamente, com 90 pontos de venda de comida de rua no Centro Histórico de Cartagena de Índias (dados ainda não publicados), foi abordado um indivíduo em cada ponto, perfazendo um total de 90 entrevistados.

Para a coleta de informações, foi utilizado um questionário contendo 23 questões, organizadas em quatro blocos: 1 . identificação do consumidor, dados sociodemográficos, 2. hábitos e motivos de consumo (principal motivo de consumo, frequência de consumo, alimentos consumidos e valor gasto em comida de rua) e 3. percepção de higiene e riscos (perspectiva nutricional, higiene dos alimentos, qualidade das frutas, risco para a saúde) e 4. Opinião.

O preenchimento dos questionários foi realizado por entrevistadores treinados e supervisionados. Como critérios de inclusão, foram considerados: indivíduos de ambos os sexos, nativos, maiores de 18 anos, que compravam e consumiam alimentos vendidos na rua e que concordaram em participar da pesquisa, voluntariamente. Foram excluídos entrevistados que não consumiam alimentos comercializados na rua, menores de 18 anos de idade e estrangeiros. Os entrevistados foram abordados em diferentes períodos no dia (manhã, horário de almoço e tarde).

O estudo foi aprovado pelo Comitê de Ética em Pesquisa com Seres Humanos da Universidade Federal da Bahia, Brasil (Parecer No. 2.464.030) e pela Vice-reitoria de investigação da Universidade de Cartagena-Colômbia (Parecer $\mathrm{N}^{\circ}$ 4351032018). A coleta de informações ocorreu durante os meses de setembro e novembro de 2018.

Para participação, os consumidores foram esclarecidos quanto aos objetivos do estudo, a metodologia e os seus direitos de proteção enquanto participantes da pesquisa. A concordância de participação voluntária foi atestada por meio de assinatura em Termo de Consentimento Livre e Esclarecido. 


\subsection{Tratamento estatístico dos dados}

Os dados foram tabulados em planilhas do Microsoft Excel, versão Windows 10 Pro e tratados por estatística descritiva e exploratória, utilizando o Software R versão 3.5.1. Foram realizados modelos de regressão logística, para determinar a probabilidade entre as variáveis independentes associadas aos motivos e à frequência de consumo, adotando-se um nível de probabilidade de 0,05 .

\section{Resultados e Discussão}

\subsection{Perfil sociodemográfico dos consumidores de comida de rua}

A Tabela 1 descreve os resultados do perfil sociodemográfico dos participantes do estudo. A maioria dos entrevistados (70\%) era do sexo masculino, com predomínio das faixas etárias até 24 anos (32,2\%) e entre 35-59 anos (31,1\%). Esses resultados concordam com os estudos realizados em outros países, evidenciando a predominância em indivíduos jovens do sexo masculino, que comem mais fora de casa, em comparação com outros grupos populacionais. Entre as justificativas para esta prática, alguns estudiosos consideram a rotina de trabalho, que poderia dificultar o preparo da comida em casa (Samapundo et al., 2015; Hiamey \& Grace, 2018). Nessa perspectiva, a comida de rua revela a sua conveniência.

Tabela 1. Perfil sociodemográfico dos consumidores de comida de rua, no Centro Histórico de Cartagena de Índias, Colômbia, 2018.

\begin{tabular}{llc}
\hline Variáveis & Categoria & Frequência $\boldsymbol{N}(\%)$ \\
\hline Gênero & Feminino & $27(30,0)$ \\
& Masculino & $63(70,0)$ \\
Faixa etária (anos) & & \\
& Até 24 & $29(32,2)$ \\
& $25-34$ & $25(27,7)$ \\
& $35-59$ & $28(31,1)$ \\
Estado civil & $>60$ & $8(8,8)$ \\
& Solteiro & $40(44,4)$ \\
& Casado & $26(28,8)$ \\
Nível de escolaridade & União estável & $20(22,2)$ \\
& Divorciado/Separado & $4(4,4)$ \\
& Até ensino fundamental completo* & \\
& & $12(13,32)$ \\
Trabalho / ocupação & Ensino médio completo/incompleto & $36(40,0)$ \\
& & $42(46,6)$ \\
Renda média mensal & Superior completo & $72(80,0)$ \\
& Sim & $18(20,0)$ \\
& Não & $39(48,7)$ \\
& $<1$ SM** & $36(45,0)$ \\
& $1-3$ SM & $4(5,0)$ \\
& $3-5$ SM & $1(1,2)$ \\
& $>5$ SM & $10(11,1)$ \\
\hline
\end{tabular}

*Incluídos nesta categoria: analfabeto, ensino fundamental incompleto e fundamental completo.

**SM= salário mínimo. Em 2018, o salário mínimo foi de US\$ 781.242 pesos colombianos (US\$237)

Fonte: Dados da pesquisa dos autores. 
No estudo de Samapundo et al. (2015), que avaliou o consumo de alimentos comercializados em Port-au-Prince, Haiti, verificou-se que, dos 160 entrevistados, a maior parte (62,5\%) era do sexo masculino e desses, 46,2\% tinham entre 15 e 25 anos. Resultado similar também foi reportado por Asiegbu et al., (2015), em trabalho realizado em Joanesburgo, África do Sul: de 402 consumidores de comida de rua entrevistados, 56,5\% eram homens e desses, $60 \%$ tinham menos de 35 anos.

Quanto ao estado civil, a maioria dos entrevistados $(44,4 \%)$ eram solteiros, seguido por casados $(28,8 \%)$ e aqueles em união estável $(22,2 \%)$. Nesse sentido, nota-se que pessoas solteiras compram mais alimentos vendidos na rua, do que aquelas de outro estado civil. As razões podem estar associadas ao fato de que as pessoas solteiras (especialmente homens) são menos propensos a cozinhar em casa, porque não têm tempo para o preparo ou, simplesmente, devido a deficiência em habilidades culinárias. Segundo Liu et al.(2014), em outros lugares, têm sido reportados que trabalhadores solteiros sentem maior necessidade de comprar alimentos vendidos na rua, devido à relutância em cozinhar, após um dia de trabalho.

$\mathrm{Na}$ análise do nível de escolaridade, verificou-se que 46,6 \% tinham completado o ensino superior e 40,0\% estavam cursando ou tinham completado o ensino médio. Cabe destacar, que a maioria dos entrevistados $(80,0 \%)$ relatou ter algum trabalho ou ocupação, dentre as ocupações encontravam-se comerciantes, trabalhadores autônomos, professionais e estudantes. Pelos achados, grande parte dos consumidores eram devidamente alfabetizados e/ou tinham uma ocupação. Assim, compuseram um grupo que, pelo perfil, se espera ser mais consciente quanto às escolhas alimentares e aos locais de obtenção. Resultados parecidos foram descritos por Samapundo et al. (2015), em Port-au-Prince, Haiti, onde a maior parte dos participantes (76\%) estava no ensino médio ou na universidade.

Estes resultados contrastam com descrição apresentada por Alimi, 2016, em estudo de revisão sobre os fatores de risco nas práticas de comida de rua, em países em desenvolvimento. Segundo o autor, a escolaridade é um dos fatores que influenciam a alimentação do consumidor e a busca da segurança, sendo os alimentos de rua consumidos principalmente por pessoas com baixo nível de educação e de renda.

Avaliando-se a renda mensal dos entrevistados, 48,7\% indicaram ganhar menos de um salário mínimo (SM), enquanto para 45,0\% o ganho variava de um a três SM por mês. Nota-se que os entrevistados estavam compreendidos em diferentes grupos de renda, com grande parte ganhando menos de um salário mínimo por mês, o que indica pertencerem aos grupos de baixa renda.

Quanto ao gasto semanal com comida de rua, entre os participantes, verificou-se que porcentagem significativa $(59,0 \%)$ informou gastar menos de COP\$20.000/semana ( $<$ US\$ 5,62), 32,9\% gastavam entre COP\$21.000 - \$60.000 (US\$ $5,90-16,85$ ), e 7,9\% gastavam mais de COP\$61.000/semana (US\$16,85).

Considerando o salário mínimo na Colômbia, em 2018 - COP 781,242 (US \$ 237,00), observou-se que os consumidores gastavam, em média, COP\$ 183.396/mês (US\$ 50,81) em comida de rua, o que corresponde a 23,5\% do SM mensal e evidencia uma relação marcante entre as condições socioeconômicas e os gastos com a comida de rua. De acordo levantamentos da Organização das Nações Unidas para Alimentação e Agricultura (FAO), para as famílias latino-americanas, $30 \%$ de suas despesas são de gastos com comida de rua, o que se considera um percentual elevado e reforça o papel deste setor para consumidores de renda baixa e média (Banco Mundial, 2018).

No estudo de Mensah et al.(2013), ao analisar o consumo de comida de rua, em quatro áreas de Kumasi, Gana, constatou-se que o maior percentual $(43,1 \%)$ dos consumidores estava compreendido no grupo de renda média (101 a 500 GHC por mês). Segundo os autores, a população em geral, gasta uma parte relevante de seu orçamento alimentar em comida de rua, destacando-se as pessoas que frequentavam os grandes centros urbanos. Deste modo, reafirma-se a aquisição desses alimentos a preços acessíveis, sobretudo para classes mais populares(Wiatrowski et al., 2021). 


\subsection{Práticas e motivos para comprar e consumir alimentos vendidos na rua}

Em relação às práticas alimentares e à formação dos hábitos, 70\% dos entrevistados informaram o consumo da comida de rua desde a infância, 25,5\% desde a adolescência/juventude, e 4,4\% na idade adulta. Este resultado evidencia a influência da própria família no consumo da comida de rua, ainda cedo, quando os pais, buscando praticidade nas compras e na elaboração das preparações, passam a oferecer às crianças estes tipos de alimentos. Assim, este hábito tende a persistir na adolescência e na idade adulta (Torres Pabón, 2019).

Estes achados se assemelham, em parte, com descrições apresentadas por Cardoso et al. (2017), que estudaram consumidores de comida de rua em Salvador, Brasil. De acordo com os autores, para 50,4\% dos participantes, o consumo da comida de rua iniciava-se na adolescência, enquanto para 32,6\% este consumo ocorria ainda na infância, o que também demonstra o hábito do consumo destes alimentos, perpetuado culturalmente pelas famílias.

Em estudo de Durán-Agüero et al. (2018), realizado em 11 países ibero-americanos (Argentina, Brasil, Chile, Colômbia, Costa Rica, Guatemala, Panamá, Paraguai, Perú, Portugal e Uruguai), com 8895 entrevistados, observou-se que jovens, entre 18 e 30 anos, apresentavam maior consumo (63,6\%), quando comparados aos maiores de 60 anos (18,4\%). Neste contexto, pontua-se que a escolha de consumo dos jovens estava associada à falta de tempo para preparar as refeições em casa e devido ao baixo custo dos alimentos.

Quanto aos motivos principais para comprar os alimentos de rua, em Cartagena, destacaram-se o fácil acesso (41,1\%), o gosto/prazer (38,3\%), falta de tempo (21,1\%) e preço (11,1\%) (Figura 1), o que evidencia a comida de rua como uma opção boa, em termos da praticidade e de questões culturais - o gosto e o prazer associado ao alimento local. Segundo Sanlier et al. (2018), a comida de rua é marcada pela variedade de sabores, baixo custo, acessibilidade e praticidade, além de ter importante papel social e na preservação do patrimônio cultural.

Em relação à frequência do consumo (Figura 2), a maior parte dos participantes $(57,7 \%)$ informou comprar e consumir comida de rua pelo menos uma vez por semana, sendo que 32,2\% faziam uso diariamente, confirmando o hábito.

Nesse contexto, verificou-se influência das variáveis sexo e escolaridade em relação aos motivos de consumo gosto/prazer e à frequência de consumo. Entre as pessoas com maior nível de educação, registrou-se que a probabilidade de consumir comida de rua por gosto/prazer foi de 0,583. Todavia, o consumo diário entre mulheres com nível educativo baixo foi maior (0,9291), em relação aos homens com níveis educativos altos $(0,0810)$. 
Figura 1. Distribuição dos participantes (\%), quanto aos motivos de consumo da comida de rua no Centro Histórico de Cartagena de Índias, Colômbia, 2018.

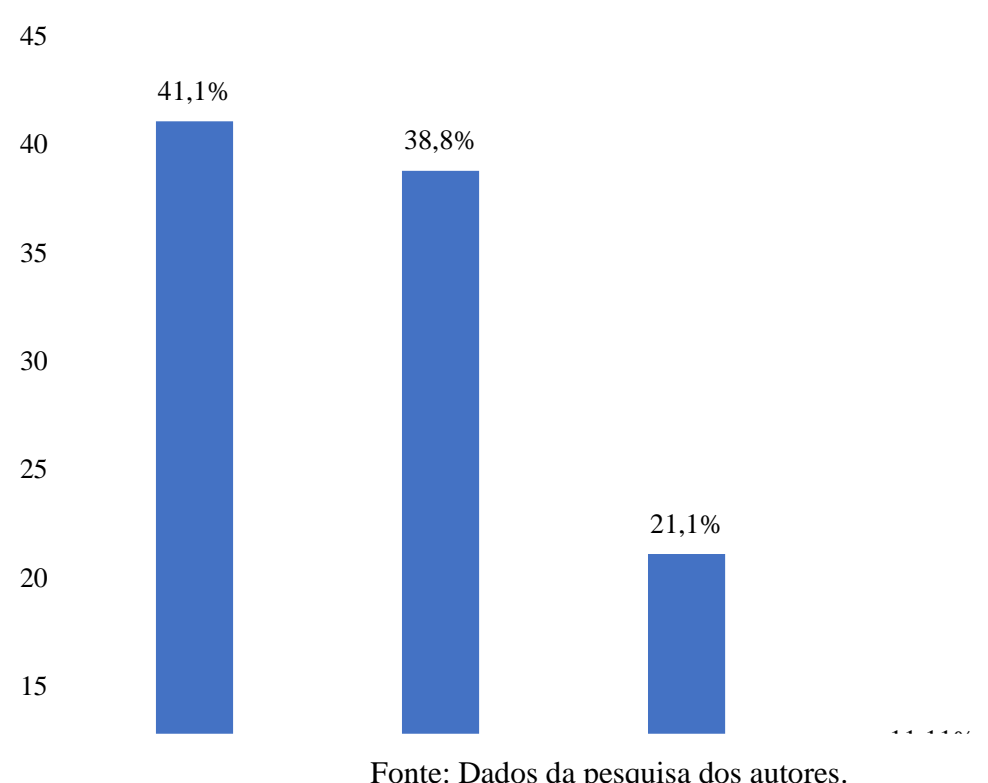

Esses resultados revelam que o nível educativo dos indivíduos influencia na frequência do consumo de comida de rua. Segundo Mensah et al. (2013), quanto maior o nível de escolaridade do consumidor, menor é o consumo de comida de rua, um quadro que pode estar associado à melhor condição de trabalho e remuneração, bem como à maior consciência dos cuidados com a saúde.

Figura 2. Distribuição dos participantes (\%), quanto à frequência de consumo da comida de rua, no Centro Histórico de Cartagena de Índias, Colômbia, 2018.

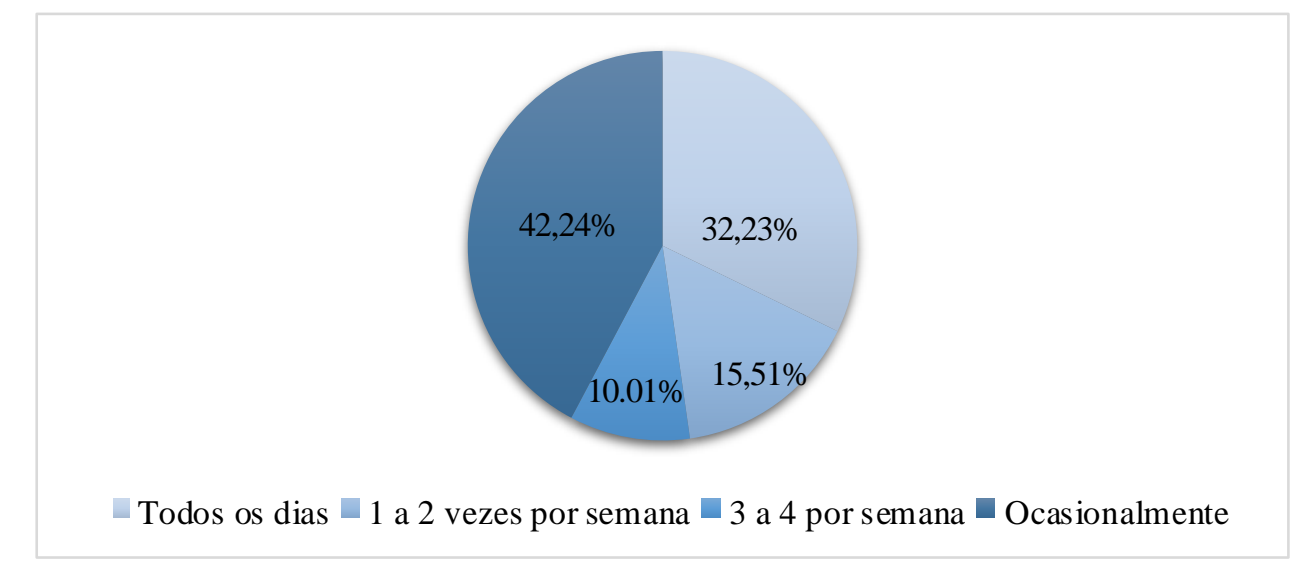

Fonte: Dados da pesquisa dos autores.

Os achados em Cartagena, em grande parte, concordam com relatos de Liu et al. (2014), em estudo com 1000 consumidores de comida de rua, em Shijiazhuang, China, onde os fatores mais importantes na decisão da compra desses alimentos foram o apelo sensorial (41\%), o baixo preço (33\%) a conveniência (15\%) e a familiaridade (8\%).

Asiegbu et al. (2016), em Joanesburgo, África do Sul, que descrevem o consumo da comida de rua junto a 402 entrevistados, reportam que, em escala semanal, 52,5\% disseram consumir frequentemente e $29,4 \%$ consumir sempre. Dentre 
os motivos mais indicados para o consumo constaram acessibilidade (51\%) e melhor sabor (35\%), o que concorda com os achados.

De igual modo, o estudo multicêntrico de Durán-Agüero et al. (2018), com 8.895 participantes, apontou que 53,6\% dos entrevistados compravam e consumiam alimentos de rua. Desses, 39,6\% consumiam porque os alimentos estavam disponíveis em locais de muito trânsito, $34,0 \%$ por serem saborosos, 14,9\% porque tinham pouco tempo para se alimentar e $11 \%$ por serem baratos. Esses resultados confirmam que a comida de rua atende a uma necessidade importante da população urbana, nos países em desenvolvimento, pela sua disponibilidade, acessibilidade e baixo custo.

Os alimentos mais consumidos pelos cartageneros foram as frituras (53,3\%), as frutas $(26,6 \%)$ e os sucos naturais $(23,3 \%)$, havendo menor consumo de produtos industrializados e outros produtos, como café e peto ou mazamorra - bebida preparada com milho branco, leite, açúcar e canela, semelhante ao mungunzá brasileiro (Figura 3). Para 93,3\% dos entrevistados, a venda de frituras e de frutas constituía parte da cultura da cidade, o que revela a oferta desses alimentos relacionada com práticas alimentares locais (Herrán et al., 2020). Quanto aos sucos, ressalta-se que, além do baixo custo e de atenderem à cultura popular, representam uma opção para saciar a sede, dadas as altas temperaturas de Cartagena, que é uma cidade tropical.

Estes resultados são similares aos descritos por Morales Pinto et al.(2019), em estudo realizado no entorno de um campus Universitário na cidade de Barranquilla/Colômbia, no qual, de 378 participantes, 67,3\% informaram consumir alimentos fritos - neste caso, porém, o consumo de frutas foi mínimo (5,8\%). Em Cartagena, apesar do consumo de alimentos ricos em carboidratos e gorduras (no caso as frituras), a oferta de frutas compreende uma opção saudável para os consumidores. Embora o relatório Panorama da Segurança Alimentar e Nutricional na América Latina e Caribe reporte baixo percentual (4 \%) de consumo de frutas e verduras, na Colômbia (WHO, 2017), pontua-se que a oferta de frutas, pelo segmento de comida de rua, representa uma prática promotora de saúde, que contribui para prevenir doenças crônicas não transmissíveis, devendo ser apoiada.

Figura 3. Distribuição (\%) dos consumidores, quanto aos tipos de alimentos mais consumidos nas ruas do Centro Histórico de Cartagena de Índias, Colômbia, 2018.

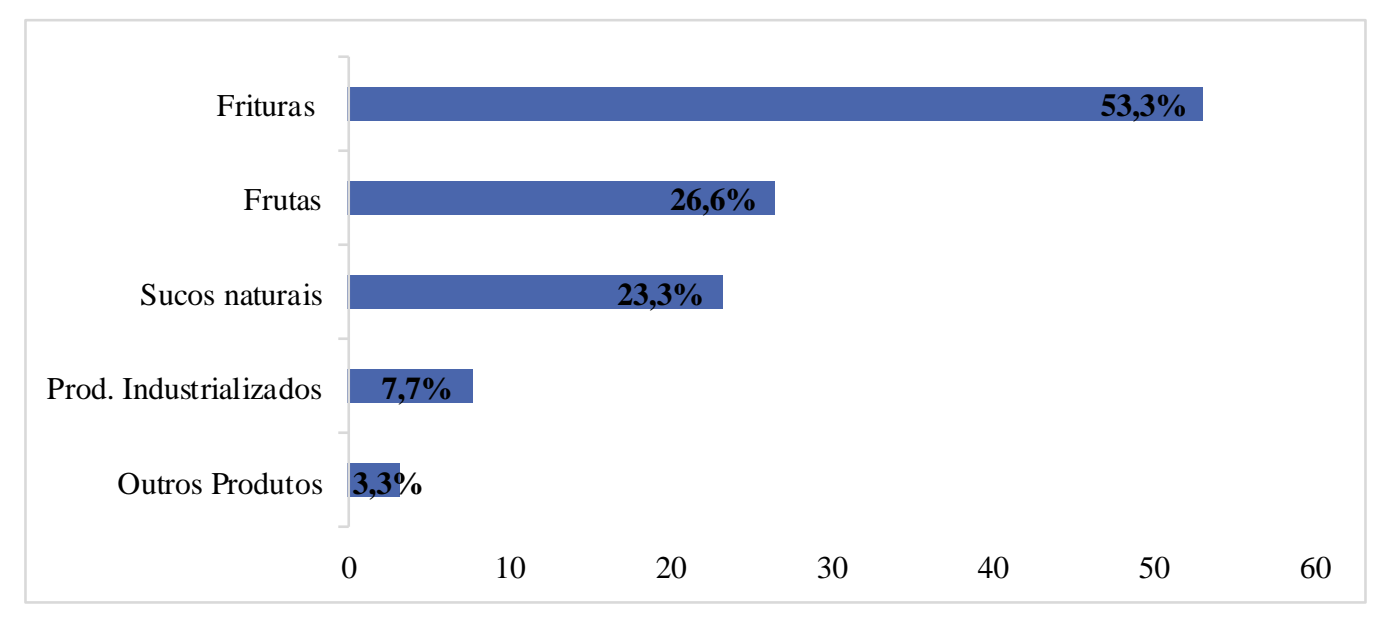

Fonte: Dados da pesquisa dos autores

Considerando os resultados do modelo de regressão, identificou-se maior probabilidade de comer mais frituras $(0,8733)$ em pessoas do sexo feminino, menores de 24 anos, que ganhavam menos de um salário mínimo, o que pode estar associado às escolhas alimentares dos jovens, que diferem muito daquelas das pessoas idosas. 
Nesse sentido, considera-se que os jovens preferem comida barata, dado que a maioria ainda é estudante e tem poder aquisitivo inferior ao de trabalhadores. Esses resultados concordam com relato de Durán-Agüero et al. (2018), que, ao comparar participantes de faixa etária distintas, constatou a escolha mais seletiva de alimentos vendidos na rua nas faixas de maior idade. No estudo de Sanlier et al. (2018), na Turquia, junto a 847 jovens consumidores de comida de rua que cursavam o ensino médio e / ou nível superior, revelou-se que o consumo de comida de rua é preferido pelos jovens, principalmente estudantes, devido ao seu sabor, acessibilidade, variedade e baixo preço.

Em Cartagena, ainda, observou-se que as variáveis que influenciaram no consumo de frutas foram o sexo e a faixa etária. Para indivíduos do sexo masculino, maiores de 60 anos, a probabilidade de comer mais frutas foi de 0,8502 , enquanto para pessoas do sexo feminino, na faixa etária de 25 a 34 anos, a probabilidade foi de 0,3630.

Quanto aos produtos industrializados, observou-se maior probabilidade do consumo entre os homens $(0,1852) \mathrm{em}$ relação às mulheres $(0,0317)$. Este achado pode estar associado ao fato de as mulheres serem mais cuidadosas com seus hábitos alimentares, devido à autoimagem e à aparência física, evitando o consumo de alimentos processados e gordurosos(Mensah et al., 2013).

Em Cartagena, ainda, constatou-se que 51,11\% dos entrevistados compravam comida de rua sempre do mesmo vendedor, sendo que $23,33 \%$ declararam facilidade na forma de pagamento - desconto e/ou crédito para compra. Esses resultados evidenciam a construção de relações socais leais e de benefício mútuo (Javela et al., 2009).

\subsection{Percepção dos consumidores em relação à saúde, higiene e riscos}

Quanto à composição nutricional da comida de rua, em Cartagena de Índias, 50\% dos consumidores informaram pensar neste atributo, apenas como fonte energética, sem considerar os fatores prejudiciais, como o elevado teor de sódio, gordura saturada e açúcares. Assim, cabe pontuar a importância dos alimentos de rua como parte do consumo alimentar da população, posto que, muitas vezes, esses alimentos servem como substitutos das principais refeições domésticas. Especialmente no centro de Cartagena, este segmento atende a estudantes, servidores públicos e cidadãos, corresponsáveis pela sustentabilidade econômica da atividade.

Steyn et al. (2014), no seu estudo de revisão que avaliou a contribuição energética da comida de rua, em países em desenvolvimento, registrou índice de atendimento que variava de $13 \%$ a 50\% dos requerimentos diários de energia, para indivíduos adultos e, de $13 \%$ a $40 \%$, para crianças. Deste modo, se confirma que esses alimentos contribuem significativamente para a dieta da população, tanto em termos de energia como também para outros nutrientes, a depender do conjunto de alimentos disponibilizado pelo segmento.

Quanto à percepção dos consumidores em relação à higiene e aos riscos associados ao comer alimentos prontos vendidos, nas ruas do Centro Histórico de Cartagena de Índias, a maior parte $(94,4 \%)$ indicou que a comida de rua poderia oferecer algum risco à saúde. Nesse sentido, considera-se que os consumidores trazem consigo algum tipo de conhecimento ou experiência prévia que lhes faz pensar sobre a insegurança desses alimentos. Ademais, entre eles, $60 \%$ disseram já ter tido indisposições de saúde, após o consumo da comida de rua.

Em estudo conduzido em Salvador, Brasil, com 1004 consumidores de comida de rua, 34,5\% dos entrevistados relacionaram o consumo de alimentos de ruas com doenças gastrintestinais entre os familiares, e, entre os participantes, 14,2\% relataram casos de doenças de origem alimentar e 13,7\% sintomas sugestivos (Cardoso et al., 2007). Estudos, em diferentes países, têm evidenciado riscos associados ao consumo de alimentos comercializados nas ruas, apontados como causadores de surtos de toxinfecção alimentar(Alimi, 2016; Kaptso et al., 2021; Wiatrowski et al., 2021)

Quanto ao sentimento associado ao consumo de comida de rua (Figura 4), considerando a saúde, 48,8\% dos consumidores em Cartagena afirmaram se sentir bem, porém 30\% afirmaram se sentir inseguros. É conhecido que a comida de 
rua pode constituir risco à saúde, devido às más condições de higiene e aos poucos recursos dos vendedores para aplicar corretamente as práticas de preparo dos alimentos (Sanlier et al., 2018).

Figura 4. Distribuição (\%) dos participantes, quanto ao sentimento associados ao consumo de comida, considerando o aspecto saúde. Cartagena de Índias, 2018.

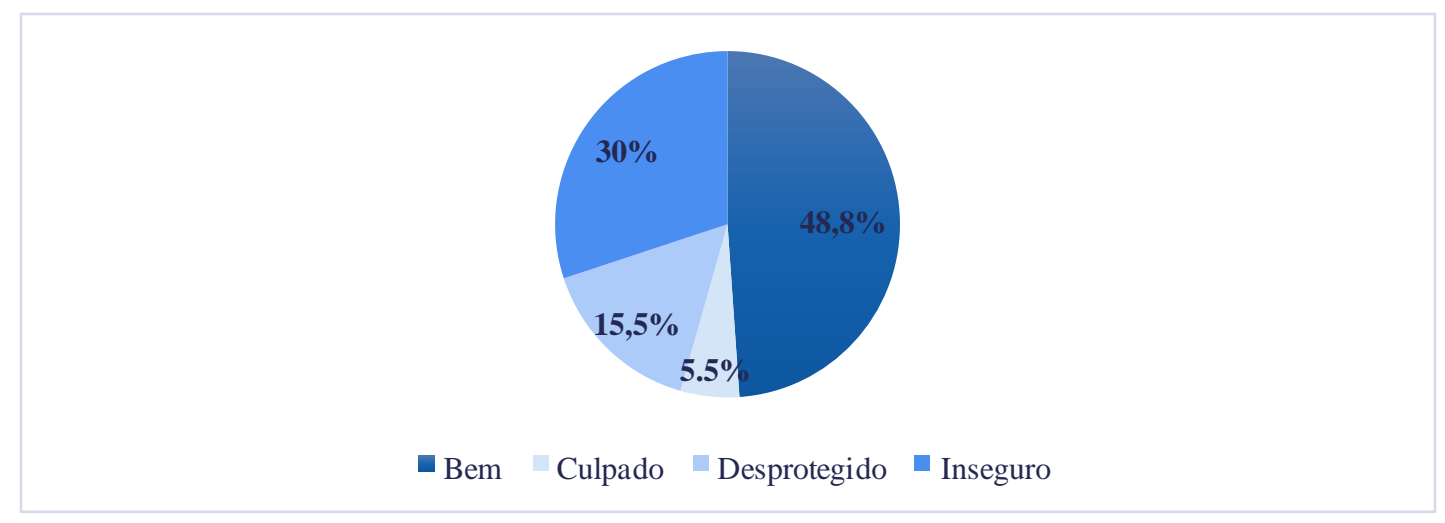

Fonte: Dados da pesquisa dos autores.

Estudos realizados em países em desenvolvimento têm avaliado a percepção dos consumidores em relação à segurança dos alimentos de rua, sendo descritas preocupações com a saúde (Alimi, 2016; Ezekiel et al., 2013; Samapundo et al., 2015). No estudo de Liu et al. (2014), realizado na Cidade de Shijiazhuang, China, com 1000 consumidores de comida de rua, apenas $18 \%$ reconheceram a comida de rua como totalmente segura. Por outro lado, no estudo de Asiegbu et al. (2016), em Johanesburgo, África do Sul, com 402 entrevistados, 58\% afirmaram estar confiantes na segurança da comida vendida na rua.

Entre os entrevistados colombianos, os pensamentos mais associados à comida de rua, no momento da aquisição, foram: a fome (42,7\%); a vontade (29,2\%); e o preço (12,3\%) - 7,8\% indicaram outros fatores na tomada de decisões, como hábito, sabor, falta de tempo, frescor dos alimentos e higiene. Pelos resultados, nota-se que a maioria dos consumidores estava mais preocupada em satisfazer as suas necessidades fisiológicas, do que com outros fatores, incluindo as práticas de manuseio dos vendedores e a segurança de alimentos - embora quase todos tivessem indicado a possibilidade de riscos à saúde.

Em uma perspectiva distinta, no estudo de Mensah et al. (2013), foram avaliados motivos que influenciavam os consumidores à não aquisição da comida de rua, sendo indicados: o ambiente insalubre (33\%), o não cumprimento das questões de higiene (limpeza) (27\%); a possibilidade de riscos à saúde / doenças associadas ao consumo de comida de rua e (25\%); e alimentos não higienizados (15\%).

Nesse contexto, é provável que algum nível de conscientização sobre as questões de segurança de alimentos, para a comida de rua, contribua para a percepção dos consumidores, com relação às práticas de segurança que influenciam na escolha do local de compra (Ezekiel et al., 2013).

Nas ruas do Centro Histórico de Cartagena, a venda de frutas é habitual e essas são comercializadas tanto inteiras como cortadas em porções. Quando cortadas, são servidas em pratos ou copos descartáveis, contudo, ficam expostas à temperatura ambiente, sem maiores cuidados de conservação e de proteção contra insetos e poeira, o que pode afetar a sua qualidade e segurança microbiológica. Mediante esta realidade, quando arguidos sobre a confiabilidade na segurança desses vegetais, para o consumo, verificou-se respostas titubeantes: 52\% dos entrevistados informaram confiar “às vezes" na qualidade e higiene das frutas vendidas na rua, ou a não confiança $(20 \%)$. 
Conforme relatado na literatura, a preocupação com a higiene e a segurança dos alimentos comercializados nas ruas é um dos problemas mais relevantes para a saúde pública. Em muitos países, entretanto, esse comércio não é regulamentado, havendo pouca atuação dos órgãos da Vigilância Sanitária, o que aumenta os riscos para os consumidores. Deste modo, tornase fundamental que as autoridades desenvolvam ações voltadas para o segmento e adotem medidas para prevenir doenças transmitidas por alimentos à população (Mensah, et al., 2013; Durán et al., 2018).

Apesar do receio descrito, na opinião dos entrevistados em Cartagena, as vendas de alimentos nas ruas eram importantes, porque geravam trabalho $(81,0 \%)$ e atendiam às necessidades dos consumidores $(15,1 \%)$ - apenas, dos entrevistados $12,2 \%$ não souberam responder.

Com base nas respostas, pode-se dizer que a geração de renda se constitui uma vantagem relevante neste comércio de alimentos, nos países em desenvolvimento, sendo reconhecida por muitas pessoas. Nas publicações, a venda da comida tem sido apontada como uma estratégia para geração de trabalho e renda, sobretudo em países marcados pela desigualdade e pela exclusão social (McKay et al., 2016; Dao Truong., 2017). Conforme descrevem Steyn et al. (2013), muitos analfabetos e pessoas desempregadas, frequentemente mulheres, acreditam que a atividade se torna uma maneira simples de ganhar dinheiro com pouco investimento de capital.

\section{Conclusão}

Em diversos países do mundo, a venda de alimentos na rua torna possível atender um grande número de pessoas que procuram por alimentos baratos e acessíveis. Na cidade de Cartagena, evidenciou-se que o consumo desses alimentos no Centro Histórico, foi predominante para adultos jovens, a maioria solteiros, que trabalhavam ou estavam cursando o ensino superior.

Uma porcentagem considerável dos entrevistados consumia comida de rua pelo menos uma vez por semana, sendo essa prática estabelecida desde a infância. Entre os motivos mais indicados para a aquisição, constaram o fácil acesso aos alimentos e a conveniência, associados à necessidade de saciar a fome ou a vontade.

As frituras e as frutas foram os alimentos mais consumidos, sendo reconhecidas como parte da cultura alimentar tradicional local. Fatores sociodemográficos como sexo, idade e escolaridade registraram influência nas práticas de consumo. Além disso, observou-se que mais da metade dos consumidores de comida de rua gastavam, em média, quase um quarto do salário mínimo da Colômbia com a comida de rua.

Grande parte dos entrevistados considerou que a comida de rua poderia oferecer riscos à saúde e informou ter tido indisposição associada ao consumo desses alimentos, observando-se também pouca confiança na higiene das frutas. Ao mesmo tempo, a maioria dos consumidores pontuou a importância do setor, pela geração de trabalho e pela oferta de alimentos e contribuição nutricional.

Considerando os resultados, a relevância da temática, cada vez mais presente, e a insuficiência de pesquisas no país, são sugeridos novos estudos, que possam aprofundar conhecimentos sobre as práticas do consumo da comida de rua e os riscos associados, bem como, apoiar a condução de estudos de intervenção, que possibilitem reflexões sobre o segmento e suas relações com o trabalho e a oferta de alimentos que busquem melhorar a compreensão de questões que estão além do campo quantitativo.

Outrossim, recomenda-se que os órgãos de saúde e/ou autoridades locais implementem estratégias de intervenção, com programas de educação, que reconheçam a importância do setor e promovam a segurança dos alimentos. Nessa direção, que busquem conscientizar aos vendedores quanto à adoção das Boas Práticas de Produção e incentivar os consumidores para práticas alimentares mais saudáveis. 


\section{Agradecimentos}

À Coordenação de Aperfeiçoamento de Pessoal de Nível Superior (CAPES), pela concessão da bolsa de estudo e aos grupos de pesquisa SACIA da UFBA- Brasil e ao grupo INCAS da UDC- Colômbia, pelo apoio e as valiosas contribuições.

\section{Referências}

Abrahale, K., Sousa, S., Albuquerque, G., Padrão, P., \& Lunet, N. (2019). Street food research worldwide: A scoping review. Journal of Human Nutrition and Dietetics, 32(2), 152-174. https://doi.org/10.1111/jhn.12604

Alimi, B. A. (2016). Risk factors in street food practices in developing countries: A review. Food Science and Human Wellness, 5(3), 141-148. https://doi.org/10.1016/j.fshw.2016.05.001

Asiegbu, C. V., Lebelo, S. C., \& Tabit, F. T. (2015). The food safety knowledge and microbial hazards awareness of consumers of ready-to-eat street-vended food. Food Control. 60, 422-429.

Ayo, S., Bonabana-Wabbi, J., \& Sserunkuuma, D. (2010). Determinants of fast food consumption in Kampala, Uganda. African Journal of Food, Agriculture, Nutrition and Development, 12, 6567-6581.

Cardoso, R. de C. V., Companion, M., \& Marras, S. R. (Orgs.). (2017). Street Food Consumers in Salvador-Bahia: Habits, Knowledge and Risk Perception. In Street food: Culture, economy, health and governance (p. 384-405). Routledge.

Contreras, C. P. A., de Cassia V C, R., da Silva, L. N. N., \& Cuello, R. E. G. (2020). Street Food, Food Safety, and Regulation: What is the Panorama in Colombia?: A Review. Journal of Food Protection, 83(8), 1345-1358. https://doi.org/10.4315/JFP-19-526

Cortese, R. D. M., Veiros, M. B., Feldman, C., \& Cavalli, S. B. (2016). Food safety and hygiene practices of vendors during the chain of street food production in Florianopolis, Brazil: A cross-sectional study. Food Control, 62, 178-186. https://doi.org/10.1016/j.foodcont.2015.10.027

Diz, M. do N.; Medeiros, C. O.; Dardin, F. D.; Bertin, R. L.; DA Costa, R. L.; Mezzomo, T. R.; Stangarlin-Fiori, L. (2020). Perfil e conhecimento em segurança dos alimentos de indivíduos que trabalham em caminhões de alimentos em uma capital no sul do Brasil. Research, Society and Development, [S. 1.], v. 9, n. 2, p. e27921963. DOI: 10.33448/rsd-v9i2.1963. Disponível em: https://rsdjournal.org/index.php/rsd/article/view/1963. Acesso em: 17 nov. 2021.

Durán-Agüero, S., Arboleda, L. M., Velásquez Vargas, J. E., Fretes Centurión, G., Gonzalez Cespedes, L. E., Rocha, A., Lima, J. P., Nessier, M. C., Raimondo, E. E., Velásquez Valderrama, Á. M., Gonzalez Sanchez, J. C., Ivankovich Guillén, S., Cordón Arrivillaga, K. R., Gonzalez Madden, E. E., Mauricio Alza, S. M., León Cáceres, J., Agazzi, B., Ortiz, A., Cabanes, D., ... Torres, J. A. (2018). Caracterización del consumo de comida callejera según edad, estudio multicéntrico. Revista Española de Nutrición Humana y Dietética, 22(3), 243. https://doi.org/10.14306/renhyd.22.3.554

Ezekiel, C. N., Sulyok, M., Babalola, D. A., Ezekiel, V. C., \& Krska, R. (2013). Incidence and consumer awareness of toxigenic Aspergillus section Flavi and aflatoxin B1 in peanut cake from Nigeria. Food Control. 30, 596-601.

FAO. (2011). Promessas e desafios do setor informal de alimentos em países em desenvolvimento. http://www.fao.org/docrep/014/a1124p/a1124p.pdf.

Herrán, O. F., Bermúdez, J. N., \& Zea, M. del P. (2020). Cambios alimentarios en Colombia; resultados de dos encuestas nacionales de nutrición, $2010-2015$. Revista de la Universidad Industrial de Santander. Salud, 52(1), 21-31. https://doi.org/10.18273/revsal.v52n1-2020004

Hiamey, S. E., \& Hiamey, G. A. (2018). Street food consumption in a Ghanaian Metropolis: The concerns determining consumption and non-consumption. Food Control, 92, 121-127. https://doi.org/10.1016/j.foodcont.2018.04.034

Hill, J., Mchiza, Z., Puoane, T., \& Steyn, N. P. (2019). Food sold by street-food vendors in Cape Town and surrounding areas: A focus on food and nutrition knowledge as well as practices related to food preparation of street-food vendors. Journal of Hunger \& Environmental Nutrition, 14(3), 401-415. https://doi.org/10.1080/19320248.2018.1434104

Kaptso KG, Tchabo, W., B, C., Gillian N, A., Amungwa, F., \& Mbofung, C. Assessment of Food Hygienic and Vending Practices among Street Food Vendors in Buea and Kumba City Council (South-West Region Cameroon). Food Sci \& Nutri Tech 2021, 6(2):000263. https://doi.org/10.23880/fsnt-16000263

Liu, Z., Zhang, G., \& Zhang, X. (2014). Urban street foods in Shijiazhuang city, China: Current status, safety practices and risk mitigating strategies, Food Control. 41, 212-218.

Marras, Stefano \& Companion, Michèle \& Cardoso, Ryzia. (2014). Street Food. Culture, Economy, Health and Governance.

Mensah, J. O., Aidoo, R., \& Teye, A. N. (2013). Analysis of street food consumption across various income groups in the Kumasi Metropolis of Ghana, International Review of Management and Business Research 2(4), 951.

Morales Pinto, N., Herrera A, R., \& Llerena C, D. (2019). Microbiological evaluation in street food outside the Universidad del Atlántico North Campus. Ciencia e Innovación en Salud. https://doi.org/10.17081/innosa.64

Nielsen, I. (2016). Nielsen Insights. In $38 \%$ de los colombianos come fuera de su hogar una o más veces a la semana. https://www.nielsen.com/co/es/insights/news/2016/38-por-ciento-de-loscolombianos-come-fuera-de-su-hogar-una-o-mas-veces-a-la-semana.html.

Samapundo, S., Climat, R., Xhaferi, R., \& Devlieghere, F. (2015). Food safety knowledge, attitudes and practices of street food vendors and consumers in Port-au-Prince, Haiti, Food Control. 50, 457-466.

Sanlier, N., Sezgin, A. C., Sahin, G., \& Yassibas, E. (2018). A study about the young consumers' consumption behaviors of street foods. Ciência \& Saúde Coletiva, 23(5), 1647-1656. https://doi.org/10.1590/1413-81232018235.17392016 
Research, Society and Development, v. 10, n. 17, e20101724245, 2021

(CC BY 4.0) | ISSN 2525-3409 | DOI: http://dx.doi.org/10.33448/rsd-v10i17.24245

Steyn, N. P., Mchiza, Z., Hill, J., Davids, Y. D., Venter, I., Hinrichsen, E., Opperman, M., Rumbelow, J., \& Jacobs, P. (2014). Nutritional contribution of street foods to the diet of people in developing countries: A systematic review. Public Health Nutrition, 17(6), 1363-1374. https://doi.org/10.1017/S1368980013001158

Torres Pabón, G. (2019). ¿En qué gastamos para comer y cuánto?: Condiciones socioeconómicas y presupuesto para el consumo de alimentos (Colombia, 1993-2014). Revista Colombiana de Sociología, 42(2), 191-228. https://doi.org/10.15446/rcs.v42n2.74809

Wiatrowski, M., Czarniecka-Skubina, E., \& Trafiałek, J. (2021). Consumer Eating Behavior and Opinions about the Food Safety of Street Food in Poland. Nutrients, 13(2), 594. https://doi.org/10.3390/nu13020594 